\title{
MicroRNA processing without Dicer
}

\author{
Anne Dueck' and Gunter Meister, 2*
}

\begin{abstract}
The canonical processing of precursor microRNAs requires the endonuclease Dicer. A recent study shows that microRNAs can be processed independently of

Dicer but instead require Argonaute 2 .
\end{abstract}

MicroRNAs (miRNAs) and their manifold functions in regulating gene expression have been the focus of intensive research over the past decade [1]. miRNAs are endogenously expressed small RNAs that undergo extensive processing and finally become part of an effector complex, known as the RNA-induced silencing complex (RISC) or miRNA-containing ribonucleoprotein (miRNP) [2,3]. miRNAs are transcribed from RNA polymerase II or, rarely, RNA polymerase III promoters, yielding primary transcripts (pri-miRNAs) that are processed by a microprocessor complex containing the RNase III enzyme Drosha. The resulting stem-loop structured miRNA precursor (pre-miRNA) is exported to the cytoplasm where another RNase III enzyme, Dicer, cleaves off the loop of the hairpin to produce a short double-stranded RNA (20 to 25 nucleotides) with a two-nucleotide overhang at the 3' end and a 5' phosphate group. One of the two RNA strands is incorporated into a RISC and acts as the functional mature miRNA. The other strand, known as the passenger strand or the star strand (miRNA*), is destabilized and removed from the cell [4].

A member of the Argonaute (Ago) protein family serves as the direct interaction partner of the miRNA within the RISC. The miRNA acts to guide the RISC to its target mRNA while the Ago protein complex represses mRNA translation or induces deadenylation-dependent mRNA decay, leading to silencing of gene expression. In a recent paper in Nature, Gregory Hannon and colleagues (Cheloufi et al.) [5], describe a novel role for an Ago protein with endonuclease activity in the processing of the mouse miRNA miR-451, which is essential for early development.

\footnotetext{
*Correspondence: gunter.meister@vkl.uni-regensburg.de

'Laboratory of RNA Biology, Max Planck Institute of Biochemistry, Am Klopferspitz

18, 82152 Martinsried, Germany

Full list of author information is available at the end of the article
}

\section{The Argonaute protein family}

Ago proteins form the core components of RISC and are key effectors of small RNA function in general [6,7]. Ago proteins are characterized by a conserved structure consisting of an amino-terminal domain, a so-called Mid domain, the PAZ (PIWI-Argonaute-Zwille) and Piwi (Pelement-induced wimpy testes) domains. The PAZ domain binds the 3 ' end of the miRNA, while the Mid domain anchors the 5 ' end. The Piwi domain has been shown to structurally resemble an RNase $\mathrm{H}$ fold, and indeed, some Ago proteins can cleave an opposing RNA strand between nucleotide position 10 and 11 of the miRNA [6,7]. In mammals, only Ago2 forms an active endonuclease, although the catalytic triad is conserved in all four members of the Ago protein family (Ago1, Ago2, Ago3, Ago4).

Although Ago2 cleaves target RNAs in RNA interference (RNAi), where artificial short interfering RNAs (siRNAs) are introduced into cells for sequence-specific knockdown of gene expression, the natural function of Ago2's catalytic activity remains elusive. It is believed that miRNAs guide all four human Ago proteins (including Ago2) to partially complementary target sites on mRNAs and that Ago proteins mediate translational repression or deadenylation-dependent mRNA decay. Ago2 does not cleave such targets because full complementarity between the miRNA and the target site on the mRNA is required for cleavage. These observations raise the intriguing question of why Ago2 has maintained the ability to cleave target RNAs, whereas the other Ago proteins have lost it during evolution.

\section{Ago2 function in early murine development}

Cheloufi et al. [5] addressed this question by taking advantage of an Ago2 knockout mouse model. They observed that Ago2-null mice die during gestation, demonstrating the requirement of Ago2 for early mouse development. Experiments using a LacZ reporter showed that Ago2 is expressed at high levels in the mouse embryo as well as in the extra-embryonic compartment such as placental tissue. Ago1, in contrast, is only present in the embryo itself.

As Ago2 is the most abundant Argonaute protein in somatic cells, Cheloufi et al. used their powerful genetic system to analyze the importance of the catalytic activity 


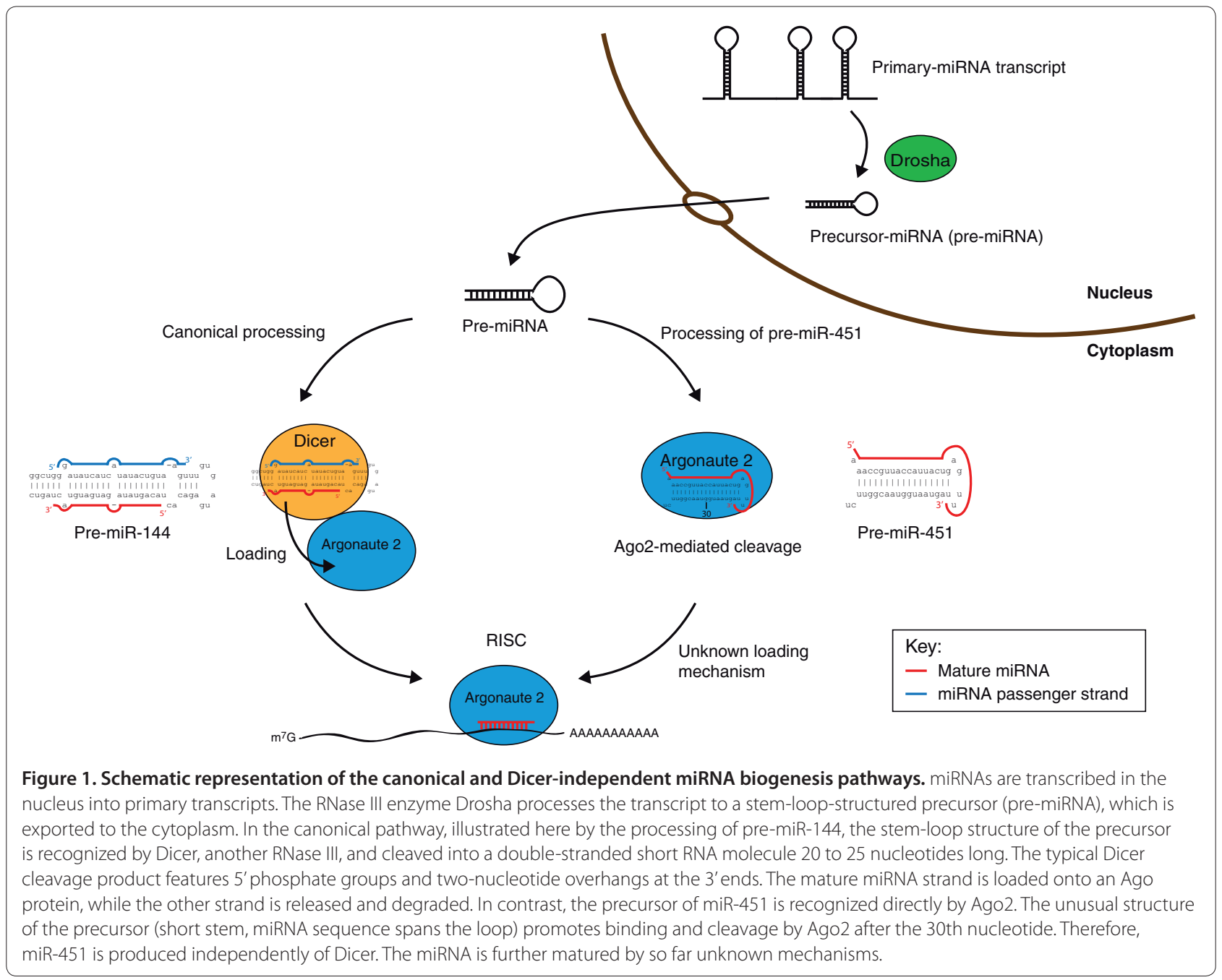

of Ago2 for mouse development [5]. They rescued the Ago2 knockout mouse by introducing a catalytically inactive Ago2 on both alleles. Homozygous mice were born according to Mendelian rules, but became pale and died a few hours after birth. The symptoms indicated severe anemia and FACS analysis revealed that the number of red blood cells in the mutant mice was decreased by $50 \%$ at birth compared to normal mice, and that the number of progenitor cells (proerythroblasts) was increased two- to threefold.

The loss of the catalytic function of Ago2 seems to severely interfere with the development and maturation of erythroblasts. This defect might be caused by a missing miRNA target cleavage and so the authors decided to profile miRNAs in wild-type and catalytically inactive Ago2 mice by deep sequencing technology [5]. Surprisingly, the expression levels of all miRNAs but one miR-451 - remained unchanged. miR-451 has already been shown to be involved in maturation of proerythroblasts into basophilic erythroblasts and is observed to decrease dramatically in mice with catalytically inactive Ago2 [8].

\section{Ago2 and miR-451 processing}

Cheloufi et al. hypothesized that the decrease in the mature miR-451 in mutant mice could be due either to processing defects or to lack of expression, and therefore analyzed miR-451 biogenesis in molecular detail. The sequence encoding miR-451 is clustered together with that for miR-144 in one gene. The authors show that no change in expression of primary transcripts occurs for either miRNA. They then transfected a DNA construct encoding the precursor of the clustered miR-144 and miR-451 into mouse embryonic fibroblasts with a conditionally expressed Drosha allele (under Cre/lox control). After loss of Drosha function by excision of the Drosha allele by Cre recombinase, the decrease in mature miR-451 was even more pronounced than the decrease in mature let-7a, a miRNA dependent on canonical processing factors (including Drosha). Therefore, Drosha is 
necessary to cleave the primary transcript of miR-451 into a precursor molecule (Figure 1).

The precursor miR-451 displays one very unusual feature. While the stem is uncommonly short (17 nucleotides), the mature miRNA (23 nucleotides) spans not only the length of the 5' arm of the stem but covers all of the loop sequence. These features are predicted to be insufficient for recognition by Dicer. The authors tested the generation of a variety of mature miRNAs from the stem-loop precursor in conditional and continuous Dicer-null mouse embryonic stem cells. In each case the levels of the mature miRNA dropped dramatically whereas no change in mature miR-451 was observed, indicating a Dicer-independent mechanism of miR-451 maturation, which had not been observed for a miRNA so far.

This left the catalytic activity of Ago2 as a candidate for processing the pre-miR-451 molecule (Figure 1). Ago2 had already been examined in terms of cleaving premiRNA rather than target mRNA. Diederichs and Haber [9] reported the cleavage of pre-let-7a in the passenger strand (the strand opposite to that of the mature miRNA) between position 10 and 11 of the sense strand. The socalled Ago2-cleaved precursor-miRNA (ac-pre-miRNA) was further processed by an unknown mechanism to yield mature let-7a. In this study, only canonical precursors with a sense and an antisense miRNA located in the stem were examined. Those precursors were all Dicer-dependent and could also be fully matured by Dicer alone.

When Cheloufi et al. [5] investigated the role of Ago2 in the processing of precursor miR-451 in the catalytically inactive Ago2 mice, Northern blot analysis revealed that the 40-nucleotide Drosha cleavage product accumulated in the mutant animals. They also found that Ago2 can bind directly to the precursor miRNA, as the 40-nucleotide cleavage product also appeared in an Ago2-targeted immunoprecipitation in the mutant cells.

If Ago2 were responsible for cleaving the miR-451 precursor, its biochemical properties would predict that it cleaved this molecule after the 30th nucleotide (Figure 1). This intermediate was looked for and found in the deep sequencing data. Here, the miR-451 read-length distribution gives a peculiar profile, with not only a sharp peak at the length of the mature miRNA, but also a second peak at longer read lengths. Examination of this intermediate showed the features of an expected Ago2cleaved product, namely a free $3^{\prime} \mathrm{OH}$ end. In contrast, Ago1 could bind the miR-451 precursor, but could not cleave it or process it into the mature form. Surprisingly, the structural features of the miR-451 hairpin could be adopted to generate other miRNAs or even siRNAs. As none of the other miRNAs in miRBase share this unusually structured hairpin, miR-451 seems to be the single exception to the canonical hairpin structure.

The study by Cheloufi et al. [5] presents a new and intriguing role for mammalian Ago2. Its essential role in cleaving the miR-451 precursor in the generation of viable mice proves the need for the evolutionary conservation of its catalytic activity. In agreement with this, the catalytic activity of Ago2 is also required for zebrafish development and cleavage of miR-451 by Ago2 is conserved between fish and mice [10].

The identification of a miRNA precursor that is bound and cleaved by Ago2 might also have an impact on the design of short hairpin RNAs (shRNAs) for gene-specific knockdown. It is tempting to speculate that such shRNAs are exclusively loaded into Ago2, and possible off-target effects caused by Ago1, Ago3, or Ago4 could be eliminated by design. Further studies will be required to determine whether or not such novel shRNAs could be useful for more specific RNAi experiments.

\section{Acknowledgements}

Our research is supported by the Max Planck Society and in part by grants from the German Bundesministerium für Bildung und Forschung (BMBF, NGFN+ \#01GS0805), the Deutsche Forschungsgemeinschaft (DFG), the Bavarian Genome Research Network (BayGene) and the European Research Council (ERC starting grant'sRNAs').

\section{Author details}

'Laboratory of RNA Biology, Max Planck Institute of Biochemistry, Am Klopferspitz 18, 82152 Martinsried, Germany. ${ }^{2}$ Universität Regensburg, Universitätsstrasse 31, 93053 Regensburg, Germany.

\section{Published: 21 June 2010}

\section{References}

1. Bushati N, Cohen SM: microRNA functions. Annu Rev Cell Dev Biol 2007, 23:175-205.

2. Carthew RW, Sontheimer EJ: Origins and mechanisms of miRNAs and siRNAs. Cell 2009, 136:642-655.

3. Meister G, Tuschl T: Mechanisms of gene silencing by double-stranded RNA. Nature 2004, 431:343-349.

4. Bartel DP: MicroRNAs: target recognition and regulatory functions. Cell 2009, 136:215-233.

5. Cheloufi S, Dos Santos CO, Chong MM, Hannon GJ: A dicer-independent miRNA biogenesis pathway that requires Ago catalysis. Nature 2010, 465:584-589.

6. Peters L, Meister G: Argonaute proteins: mediators of RNA silencing. Mol Cell 2007, 26:611-623.

7. Hock J, Meister G: The Argonaute protein family. Genome Bio/ 2008, 9:210.

8. Papapetrou EP, Korkola JE, Sadelain M: A genetic strategy for single and combinatorial analysis of miRNA function in mammalian hematopoietic stem cells. Stem Cells 2010, 28:287-296.

9. Diederichs S, Haber DA: Dual role for argonautes in microRNA processing and posttranscriptional regulation of microRNA expression. Cell 2007, 131:1097-1108.

10. Cifuentes D, Xue H, Taylor DW, Patnode H, Mishima Y, Cheloufi S, Ma E, Mane S, Hannon GJ, Lawson N, Wolfe S, Giraldez AJ: A novel miRNA processing pathway independent of Dicer requires Argonaute2 catalytic activity. Science 2010, in press.

doi:10.1186/gb-2010-11-6-123

Cite this article as: Dueck A, Meister G: MicroRNA processing without Dicer Genome Biology 2010, 11:123. 